\title{
Postoperative Comparison In Open Vs. Laparoscopic Ventral Hernia Repair In Obese Patients
}

${ }^{1}$ 2nd Surgery Department, Emergency County Hospital, Constanta

${ }^{2}$ University "Ovidius" of Constanta, Faculty of Medicine

${ }^{3}$ Surgery Department, Ovidius Clinical Hospital, Constanta

\begin{abstract}
Introduction: Obesity is an important problem in our society. Recent studies shows that laparoscopic ventral hernia repair has advantages in obese patients comparing with the standard open approach. This study wants to compare length of stay (LOS), hospitalization costs (HC) and operative time (OT) in laparoscopic and open ventral hernia repair.

Methods: A retrospective study of obese patients $\left(\mathrm{BMI}>30 \mathrm{~kg} / \mathrm{m}^{2}\right)$ that underwent ventral hernia repair between 2014 and 2015 were included. We compared demographics, hernia size, OT, LOS, HC between the two approaches.

Results: 100 patients with ventral hernia repair were included, 11 had laparoscopic approach (11\%). Laparoscopy was performed only in elective surgery and for small defects. The operation time was almost the same (119.09 vs. $118.87 \mathrm{~min})$. The length of stay was significantly longer in open approach ( 8.53 vs. 2.9 days) and hospitalization costs were higher for laparoscopic repair.

Conclusions: Laparoscopy offers a better length of stay, but the costs remains higher for this approach.
\end{abstract}

Keywords: obesity, ventral hernia repair, laparoscopy

Iuliana Dogaru

Surgery Department, Emergency County Hospital Constanta No 145, Tomis bldv, 900591, Constanta, Romania

email : juliadogaru@gmail.com

phone: +40724357994

\section{Introduction}

Obesity is a medical condition characterized by excess body fat with negative effects on health. It is defined as an increased body mass index (BMI $>30 \mathrm{~kg} /$ $\left.\mathrm{m}^{2}\right)$.

Obesity it is associated with high risk of cardiac and respiratory disease, diabetes mellitus and endocrine disorders. It is correlated with increased intra-abdominal pressure, having high risk of ventral hernia occurrence and complications [1].

Ventral hernia repair is one of the most common surgical intervention performed in a Surgery Department. It is classified (European Hernia Society) in primary hernias and incisional hernias [2].

Ventral hernia can be repaired using open or laparoscopic techniques.

In the last years the mini-invasive surgery of parietal defects has become popular and there is an important interest for patients and surgeons in using it. Even if for cholecystectomy laparoscopy has become the golden standard, for parietal surgery it is still limited, because of costs and difficulty in achieving surgical skills.

Some studies showed that laparoscopic repair has advantages in length of hospital stay, postoperative pain and social reinsertion, but the costs are higher than in the open surgery [3].

We present a short term comparison between 
open and laparoscopic ventral hernia repair in obese patients, unique in our country comparing the operative time, length of hospitalization and costs. fascia. The mesh is fixed with non-absorbable sutures. All the hernia repair with mesh placement respect the principle of "tension-free technique".

\section{Patient selection:}

We included in our study 100 obese patients who underwent ventral hernia repair in two hospitals in our city, Emergency County Hospital Constanta and Ovidius Clinical Hospital between 2014 and 2015. All the patients included in the study had a body mass index (BMI) greater than $30 \mathrm{~kg} / \mathrm{m}^{2}$.

\section{Data collection:}

Data collection included age, sex, body mass index (BMI), type of hernia, location of the hernia, size of the defect, duration of the surgical intervention, length of hospital stay and costs.

\section{Operative technique:}

Laparoscopic approach:

The pneumoperitoneum is created using Veress needle inserted in the Palmer's point (left subcostal space) or open technique. First we place a $10 \mathrm{~mm}$ trocar in the left flank for a $30^{\circ}$ laparoscope. After abdominal inspection we insert under direct vision two other trocars $(5 \mathrm{~mm})$ in the upper and lower left quadrant.

We reduce the content of hernia, using or not monopolar diathermy and inspect the parietal defect. We never resect the peritoneal sac.

The composite mesh is placed intraabdominally, overlapping the defect at least $3 \mathrm{~cm}$ in all directions and is doubled fixed using non-absorbable sutures and tacks (titanium or absorbable).

Open approach:

We use longitudinal skin incision, hernia sac dissection, freeing the contents and sac resection. The parietal reconstruction is made with or without a mesh placement, depending on intraoperative findings and surgeon preferences. Normally we use a polypropylene mesh, placed either on-lay, after closure of the fascia or pro-peritoneal, underneath the

\section{Results}

Between January 2014 and December 2015 100 obese patients $\left(\mathrm{BMI}>30 \mathrm{~kg} / \mathrm{m}^{2}\right)$ underwent ventral hernia repair. There were 11 who had laparoscopic approach and 89 who had open approach (Figure 1). There were no conversion from mini-invasive to open technique.

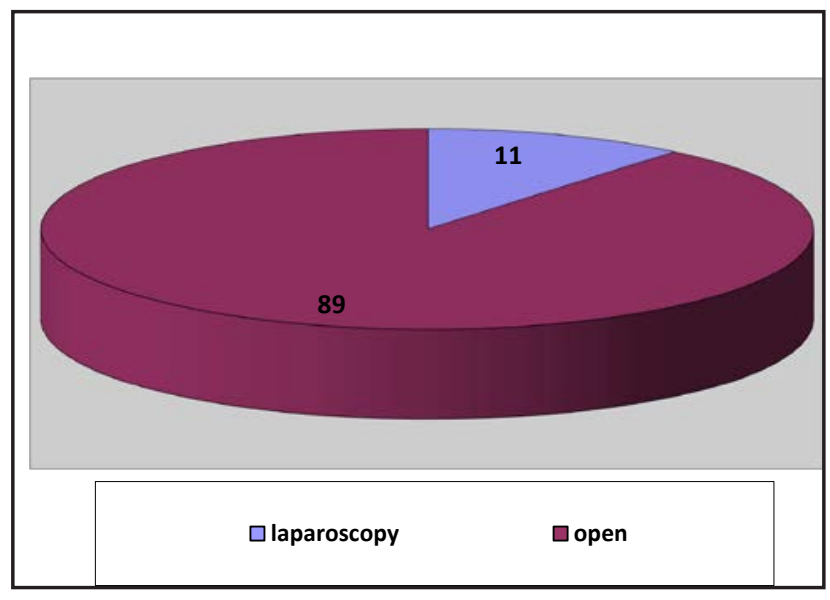

Figure 1 Operative technique

The demographic data of patients are listed in Table I.

Table I: Demographic data

\begin{tabular}{|l|c|c|}
\hline & Laparoscopic (11) & Open (67) \\
\hline Mean age (years) & 48 & 61 \\
\hline Sex (male) & 7 & 27 \\
\hline Mean BMI (kg/m $\left.\mathbf{m}^{\mathbf{2}}\right)$ & 35.33 & 34.82 \\
\hline
\end{tabular}

The perioperative data are shown in Table II. 
Table II: Perioperative data

\begin{tabular}{|l|c|c|}
\hline & Laparoscopic (11) & Open (89) \\
\hline Primary & 6 & 38 \\
\hline Incisional & 5 & 51 \\
\hline Admission & 11 & 63 \\
\hline Elective & 0 & 26 \\
\hline Emergency & 119.09 & 118.87 \\
\hline $\begin{array}{l}\text { Operation time } \\
\text { (minute) }\end{array}$ & 2.9 & 8.53 \\
\hline $\begin{array}{l}\text { Length of stay } \\
\text { (days) }\end{array}$ & 0 & 7 \\
\hline Wound infection & \multicolumn{1}{|c|}{0} \\
\hline
\end{tabular}

The hernia group was divided in ventral hernia group (44 patients) and incisional hernia group (56 patients). The patients were operated in elective surgery in 74 cases and in emergency in 26 cases. All the patients who underwent laparoscopic ventral hernia repair were elective.

The hernias were divided in small $(<5 \mathrm{~cm})$, medium $(5-15 \mathrm{~cm})$ and large $(>15 \mathrm{~cm})$. In the laparoscopic group (LVHR) were small and medium hernias, while in the open group (OVHR) all the large hernias were distributed.

The localization of the defects was in $94 \%$ in the midline, only 6 cases were situated para-rectal (1 case), in the right iliac fossa ( 1 case) and subcostal (4 cases).

The localization of the hernia is described in Figure 2.

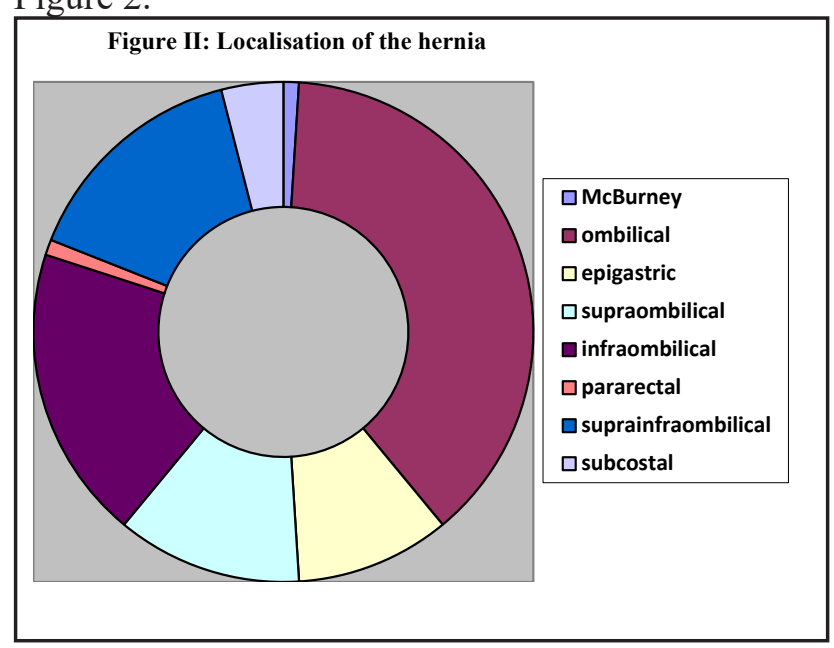

Figure 2 localization of the hernia
In the laparoscopic group all the patients had general anesthesia, while in the open repair group regional anesthesia was performed in $13.48 \%$ and general anesthesia in $86.52 \%$.

The mean operation time was almost the same in the two groups (119 minutes in LVHR versus 118 minutes in OVHR).

Wound infections were found only in the open repair group (7 patients), seroma in $18.18 \%$ of LVHR and $17.97 \%$ of OVHR.

The mean length of stay was 2.9 days for miniinvasive surgery and 8.53 days for open technique. The smaller LOS was 1 day (LVHR) and the larger was 27 days (OVHR).

The mean costs were in the LHVR (5793.88RON) versus OVHR (4634.94RON).

\section{Discussions}

In the past, obesity was considered a contraindication for laparoscopic approach, because obese patients have a high risk of respiratory and cardiac disease [4].

Nowadays, laparoscopy is a safer procedure for obese and seems to have low perioperative morbidity and low length of hospital stay $[1,4]$.

Laparoscopy was performed only in elective surgery and for small defects. The surgeons need to improve their surgical skills in the laparoscopic repair of the parietal defects.

The operation time was almost the same (119.09 vs. $118.87 \mathrm{~min}$ ).

The length of stay was significantly longer in open approach (8.53 vs. 2.9 days) and was associated with higher morbidity and mortality.

The hospitalization costs were higher for laparoscopic repair, because we need composite mesh and endotackers.

In our cohort wound infections were found only in open repair compared with laparoscopic repair. 


\section{Conclusions:}

Laparoscopy offers a better length of stay, but the costs remains higher for this approach. patient populations. Surg Endosc, 22(10), 22442250. doi: 10.1007/s00464-008-0039-1

4. Birgisson, G., Park, A.E., Mastrangelo, M.J., Jr., Witzke, D.B. \& Chu, U.B. (2001). Obesity and laparoscopic repair of ventral hernias. Surg Endosc, 15(12), 1419-1422. doi: 10.1007/ s004640080077

\section{Conflicts of interest:}

None.

\section{Acknowledgements:}

We thank to all the medical teams involved in this work.

\section{References:}

1. Froylich, D., Segal, M., Weinstein, A., Hatib, K., Shiloni, E. \& Hazzan, D. (2016). Laparoscopic versus open ventral hernia repair in obese patients: a long-term follow-up. Surg Endosc, 30(2), 670675. doi: 10.1007/s00464-015-4258-y

2. $\quad$ Liang, M.K., Berger, R.L., Li, L.T., Davila, J.A., Hicks, S.C. \& Kao, L.S. (2013). Outcomes of laparoscopic vs open repair of primary ventral hernias. JAMA Surg, 148(11), 1043-1048. doi: 10.1001/jamasurg.2013.3587

3. Ching, S.S., Sarela, A.I., Dexter, S.P., Hayden, J.D. \& McMahon, M.J. (2008). Comparison of early outcomes for laparoscopic ventral hernia repair between nonobese and morbidly obese 